\section{CAPNETZ fokussiert auf COVID-19}

CAPNETZ - Netzwerk zur Erforschung der ambulant erworbenen Pneumonie dokumentiert erste Corona-Patienten in seiner klinischen Studie

Die neuartige Lungenerkrankung COVID19 verursacht schwere klinische und intensiv-pflichtige Verläufe. Eine spezifische Therapie oder ein Impfstoff sind noch nicht in Aussicht. Neben den wichtigen allgemeinen Maßnahmen, die jüngst eingeleitet und umgesetzt werden, kommt der Erforschung der Erkrankung eine zentrale Rolle zu. Es handelt sich um eine gänzlich neuartige Form der Lungenentzündung, über die wir aktuell zu wenig wissen. Es ist unklar, welche Faktoren einen schweren Verlauf vorhersagen, welche Grunderkrankungen für einen schweren Verlauf prädisponieren, welche Komplikationen berücksichtigt werden müssen, welchen Einfluss vorbestehende Therapien haben und was die Sterblichkeit im Einzelfall bedingt. Gilt unser bisheriges Wissen über die Lungenentzündung und das Lungenversagen auch für COVID-19? CAPNETZ beabsichtigt, diese Fragen und viele mehr zu beantworten.

CAPNETZ hat sich seit 2001 zum größten Forschungsnetzwerk für die ambulant erworbene Pneumonie weltweit entwickelt. Von über 13000 Patienten konnte CAPNETZ klinische Daten in seiner Beobachtungsstudie zur Lungenerkrankung dokumentieren, welche detaillierte Analysen in der Forschung erlauben. Die Patienten werden wissenschaftlich hinsichtlich zugrunde liegender Erreger, Therapiemanagement, Begleiterkrankungen, und des klinischen Verlaufs sowie der Sterblichkeit dokumentiert.
Nun rückte CAPNETZ die Erforschung der Corona-Erkrankung in den Fokus. Innerhalb einer Woche wurden Voraussetzungen geschaffen, um der Forschung Patientendaten zur Verfügung zu stellen. Hierbei werden aus den behandelnden Kliniken COVID-19-Patienten mit und ohne Lungenentzündung eingeschlossen. Weitere Informationen unter: www. capnetz.de/html/capnetz/project

Grit Barten-Neiner, Hannover 